ARTICLE

DOI: $10.1038 /$ s41467-018-03905-6

\title{
Pharmacodynamics of mutant-IDH1 inhibitors in glioma patients probed by in vivo 3D MRS imaging of 2-hydroxyglutarate
}

Ovidiu C. Andronesi ${ }^{1}$, Isabel C. Arrillaga-Romany ${ }^{2}$, K. Ina Ly², Wolfgang Bogner ${ }^{3}$, Eva M. Ratai ${ }^{1}$, Kara Reitz ${ }^{4}$, A. John lafrate ${ }^{5}$, Jorg Dietrich ${ }^{2}$, Elizabeth R. Gerstner ${ }^{2}$, Andrew S. Chi ${ }^{6}$, Bruce R. Rosen ${ }^{1}$, Patrick Y. Wen ${ }^{7}$, Daniel P. Cahill ${ }^{4} \&$ Tracy T. Batchelor ${ }^{2}$

Inhibitors of the mutant isocitrate dehydrogenase 1 (IDH1) entered recently in clinical trials for glioma treatment. Mutant IDH1 produces high levels of 2-hydroxyglurate (2HG), thought to initiate oncogenesis through epigenetic modifications of gene expression. In this study, we show the initial evidence of the pharmacodynamics of a new mutant IDH1 inhibitor in glioma patients, using non-invasive 3D MR spectroscopic imaging of 2HG. Our results from a Phase 1 clinical trial indicate a rapid decrease of $2 \mathrm{HG}$ levels by $70 \%(\mathrm{Cl} 13 \%, P=0.019)$ after 1 week of treatment. Importantly, inhibition of mutant IDH1 may lead to the reprogramming of tumor metabolism, suggested by simultaneous changes in glutathione, glutamine, glutamate, and lactate. An inverse correlation between metabolic changes and diffusion MRI indicates an effect on the tumor-cell density. We demonstrate a feasible radiopharmacodynamics approach to support the rapid clinical translation of rationally designed drugs targeting IDHT/ 2 mutations for personalized and precision medicine of glioma patients.

\footnotetext{
${ }^{1}$ Department of Radiology, Massachusetts General Hospital, Athinoula A. Martinos Center for Biomedical Imaging, Harvard Medical School, Boston, MA 02129, USA. ${ }^{2}$ Department of Neurology, Massachusetts General Hospital, Stephen E. and Catherine Pappas Center for Neuro-Oncology, Division of Hematology/Oncology, Harvard Medical School, Boston, MA 02114, USA. ${ }^{3}$ Department of Biomedical Imaging and Image-guided Therapy, High Field MR Centre, Medical University of Vienna, Vienna 1090, Austria. ${ }^{4}$ Department of Neurosurgery, Massachusetts General Hospital, Harvard Medical School, Boston, MA 02114, USA. ${ }^{5}$ Department of Pathology, Massachusetts General Hospital, Center for Integrated Diagnostics, Harvard Medical School, Boston, MA 02114, USA. ${ }^{6}$ Brain Tumor Center, Laura and Isaac Perlmutter Cancer Center, New York University Langone Medical Center and School of Medicine, New York, NY 10016, USA. 7 Dana-Farber Cancer Institute, Boston, MA 02284, USA. Correspondence and requests for materials should be addressed to O.C.A. (email: oandronesi@mgh.harvard.edu)
} 
ecurrent heterozygous mutations in the isocitrate dehydrogenase 1 (IDH1) enzyme are very common in grade IIIII glioma and secondary glioblastoma ${ }^{1,2}$, which now are recognized in the 2016 World Health Organization classification of central nervous system tumors ${ }^{3}$ to be a particular subtype of glioma characterized by the distinct molecular and clinical phenotypes. The metabolic hallmark of this glioma subtype is de novo production of high levels of the oncometabolite 2hydroxyglutarate $(2 \mathrm{HG})$, exclusively in the form of the $\mathrm{D}$ enantiomer (D-2HG), by the mutant IDH1 enzyme through the $\mathrm{NADPH}$-dependent reduction of a-ketoglutarate $(\mathrm{\alpha KG})^{4}$. IDH1 mutation is an early genetic event in gliomagenesis, and $2 \mathrm{HG}$ is believed to further promote tumorigenesis through the inhibition of $a \mathrm{KG}$-dependent dioxygenases ${ }^{5}$ and chromatin modifiers, resulting in DNA-/histone-hypermethylation and loss of the epigenetic control of gene expression ${ }^{6-10}$.

Gliomas are rarely curable tumors with a low survival rate (34\%) at 5 years (SEER, CBTRUS 2012). Although the mutant IDH1 glioma are more amenable to gross-total resection ${ }^{11}$ and seem to respond better to standard chemoradiation ${ }^{12-14}$ especially when associated $1 \mathrm{p} / 19 \mathrm{q}$ co-deletion, the $I D H 1$ mutations represent a clear opportunity for more targeted treatment either by small-molecule inhibitors of the mutant IDH1 enzyme ${ }^{15}$, immunotherapy ${ }^{16}$, or by synthetic lethality strategies ${ }^{17,18}$. Recently, mutant IDH1 targeted therapeutic strategies have entered Phase I clinical trials, and neuroimaging can accelerate the clinical translation of these treatments ${ }^{19-21}$. Preliminary data from the clinical trials in acute myeloid leukemia (AML) suggest that there is benefit of mutant $I D H 2$ inhibition and lowering of $2 \mathrm{HG}$ concentration ${ }^{22}$, however no data are yet available from clinical trials in mutant IDH1 glioma patients. Our study sheds light on the metabolic effects in response to mutant IDH1 inhibition in glioma patients.

The unique biology of $2 \mathrm{HG}$ makes this metabolite a very specific biomarker that can be used for the diagnostic, prognostic, prediction, and pharmacodynamics assessment by probing the tumor burden, cancer pathways, and treatment mechanisms in the mutant $I D H 1$ gliomas. $2 \mathrm{HG}$ can be detected noninvasively by in vivo magnetic resonance spectroscopy (MRS), and several methods ${ }^{23-26}$ have been demonstrated to resolve the spectral overlap between $2 \mathrm{HG}$ and other normally occurring brain metabolites. In particular, for monitoring the treatment in mutant IDH1 glioma patients the non-invasive MRS detection of $2 \mathrm{HG}$ is more feasible ${ }^{27,28}$ and has clear advantages, compared to biopsies: (1) there are no associated risks, (2) the technique can be repeated multiple times, (3) the technique can probe multiple tumor regions, and (4) MRS can investigate normal appearing brain as internal control. Alternative methods ${ }^{29-33}$, such as measuring $2 \mathrm{HG}$ in blood, urine, and CSF samples have shown mixed results for mutant $I D H 1$ glioma, with some studies reporting elevated $2 \mathrm{HG}$ only in $\mathrm{CSF}^{33}$, while others found elevated $2 \mathrm{HG}$ only in urine $^{32}$. The lack of uniform results may be related to lack of a standard protocol with differences in analytical methods, sample collection, and preservation that add to the biological variability. In addition, $2 \mathrm{HG}$ levels in periphery are diluted, the spatial localization is lost, tumor heterogeneity cannot be probed, and collecting CSF is not without complication, especially for longitudinal monitoring. Besides tumor production, $2 \mathrm{HG}$ levels in serum and urine are also influenced by other factors, such as the blood-brain barrier (BBB), which is less compromised in mutant IDH1 glioma, and the shedding of tumor material may thus be reduced. The combination of all these factors make the detection of $2 \mathrm{HG}$ in CSF, serum, and urine less straightforward in mutant IDH glioma patients, compared to AML patients. On the other hand, MRS methods are rapid, easy to perform, and inexpensive, relative to genomics or other in vivo molecular imaging, such as PET or SPECT. 2HG imaging provides better specificity for detection of IDH1 mutations than alternative MRI methods ${ }^{34-39}$, and could help distinguish true/pseudo-response in treatment assessment ${ }^{40}$. In addition, in the case of mutant IDH1 inhibitors, $2 \mathrm{HG}$ as a direct pharmacodynamic biomarker is expected to probe earliest the target modulation, compared to either conventional anatomical magnetic resonance imaging, such as contrast enhanced T1-weigted and fluid attenuated inversion recovery (FLAIR) MRI that are part of RANO criteria ${ }^{41,42}$ or the more advanced diffusion/perfusion $\mathrm{MRI}^{43}$.

In this study we used a recently demonstrated 3D MRS imaging (MRSI) method for $2 \mathrm{HG}$ detection ${ }^{27}$ to assess the pharmacodynamic effects of the new investigational drug IDH305 (Novartis Pharmaceuticals) in mutant IDH1 glioma patients enrolled in an open label first-in-human Phase I clinical trial (ClinicalTrials.gov identifier: NCT02381886). IDH305 is an orally available, brain penetrant, mutant-selective allosteric high affinity IDH1 inhibitor that acts on both canonical $(\mathrm{R} 132 \mathrm{H})$ and noncanonical (R132C) mutated enzymes, but has much lower affinity for wild-type IDH1 or mutant IDH2 enzymes. IDH305 potently reduces the $2 \mathrm{HG}$ production in preclinical models, in which a single dose of $100 \mathrm{mg} / \mathrm{kg}$ is sufficient to reduce the tumor $2 \mathrm{HG}$ levels by $95 \%$ in nude mice with IDH1 mutant flank tumors, and has shown in vitro antiproliferative effects (Novartis Pharmaceuticals Phase I clinical trial protocol, ClinicalTrials.gov identifier: NCT02381886). Hence, the biological effects of IDH305 in patients are expected to be restricted to tumor cells harboring IDH1-R132 mutations. Here, we show initial evidence regarding the pharmacodynamic effects of mutant IDH1 inhibition in glioma patients. Our primary goal in this study was to probe whether IDH305 can modulate target activity in glioma patients. In addition to $2 \mathrm{HG}$, we measured other metabolites that are

Table 1 Demographic, histology and molecular markers of patients

\begin{tabular}{|c|c|c|c|c|c|c|c|c|c|c|}
\hline Pt & $\mathbf{A} / \mathbf{G}$ & Grade & Type & IDH1 & IDH305 & $1 p / 19 q$ & TP53 & MGMT & FLAIR $\left(\mathrm{cm}^{3}\right)$ & 2HG (mM) \\
\hline$\# 1$ & $49 / F$ & III & AOD & $\mathrm{R} 132 \mathrm{H}$ & $550 \mathrm{mg}$ & co-del & n.a. & n.a. & 15.9 & 2.32 \\
\hline \#2 & $48 / F$ & II & $A$ & $\mathrm{R} 132 \mathrm{H}$ & $550 \mathrm{mg}$ & n.d. & mut & n.a. & 23.7 & 0.28 \\
\hline \#3 & $30 / F$ & IV & GBM & $\mathrm{R} 132 \mathrm{H}$ & $550 \mathrm{mg}$ & co-del & n.a. & met & 7.9 & 1.73 \\
\hline \#4 & $42 / M$ & III & $A A$ & $\mathrm{R} 132 \mathrm{H}$ & $550 \mathrm{mg}$ & not-del & mut & umet & 10.3 & 0.59 \\
\hline \#5 & $38 / M$ & III & $A A$ & $\mathrm{R} 132 \mathrm{H}$ & $550 \mathrm{mg}$ & not-del & mut & umet & 8.8 & 1.04 \\
\hline \#6 & $49 / F$ & III & $A A$ & $\mathrm{R} 132 \mathrm{H}$ & $550 \mathrm{mg}$ & co-del & n.a. & n.a. & 43.2 & 1.31 \\
\hline \#7 & $31 / M$ & III & $A A$ & $\mathrm{R} 132 \mathrm{H}$ & 900 mg & not-del & mut & n.a. & 3.9 & n.d. \\
\hline \#8 & $46 / F$ & II & $A^{\star}$ & $\mathrm{R} 132 \mathrm{H}$ & $550 \mathrm{mg}$ & n.d. & n.a. & n.a. & 7.8 & n.d. \\
\hline
\end{tabular}

Abbreviations: Pt Patient number, A/G Age/Gender, A Astrocytoma, AA Anaplastic Astrocytoma, AOD Anaplastic Oligodendroglioma, GBM Glioblastoma, IDH1 Isocitrate Dehydrogenase 1, R132H Arginine 132 to Histidine, IDH305 inhibitor of IDH1-R132H, 1p/19q co-deletion of 1p and 19q chromosome arms, TP53 Tumor Protein p53, MGMTO6 - Methylguanine DNA Methyltransferase, FLAIR Fluid Attenuated Inversion Recovery, 2HG 2-hydroxyglutarate, co-del co-delited, mut mutated, met methylated, umet un-methylated, n.a.not available, n.d. not detectable. Patients 1-5 had baseline and follow-up MRSI scans, patient 6 had only baseline scan, and patients 7-8 had no detectable $2 \mathrm{HG}$ at baseline. ${ }^{\star}$ According to WHO 2007 this patient was diagnosed as ganglioglioma with atypical features. 
$\mathbf{a}$
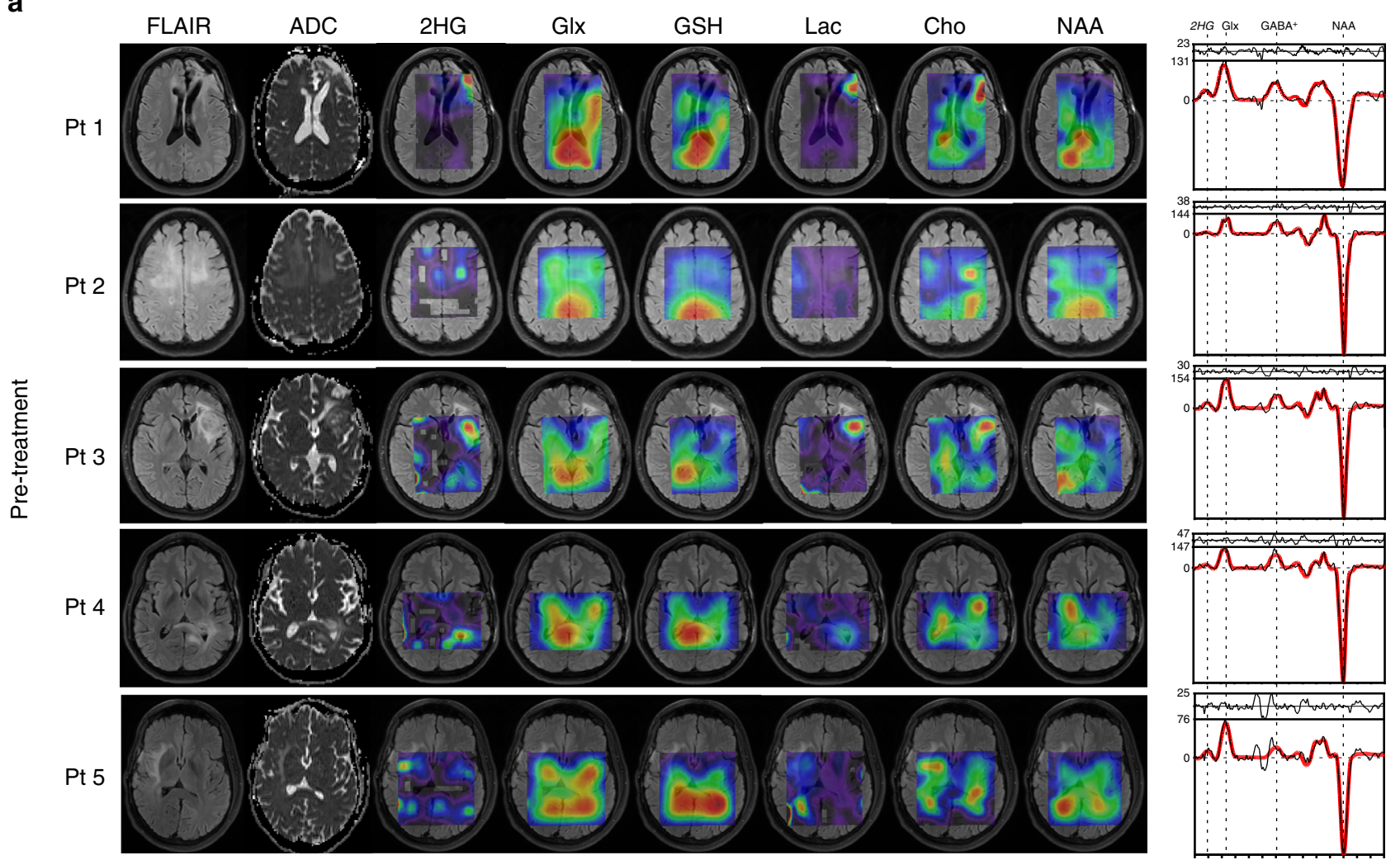

b
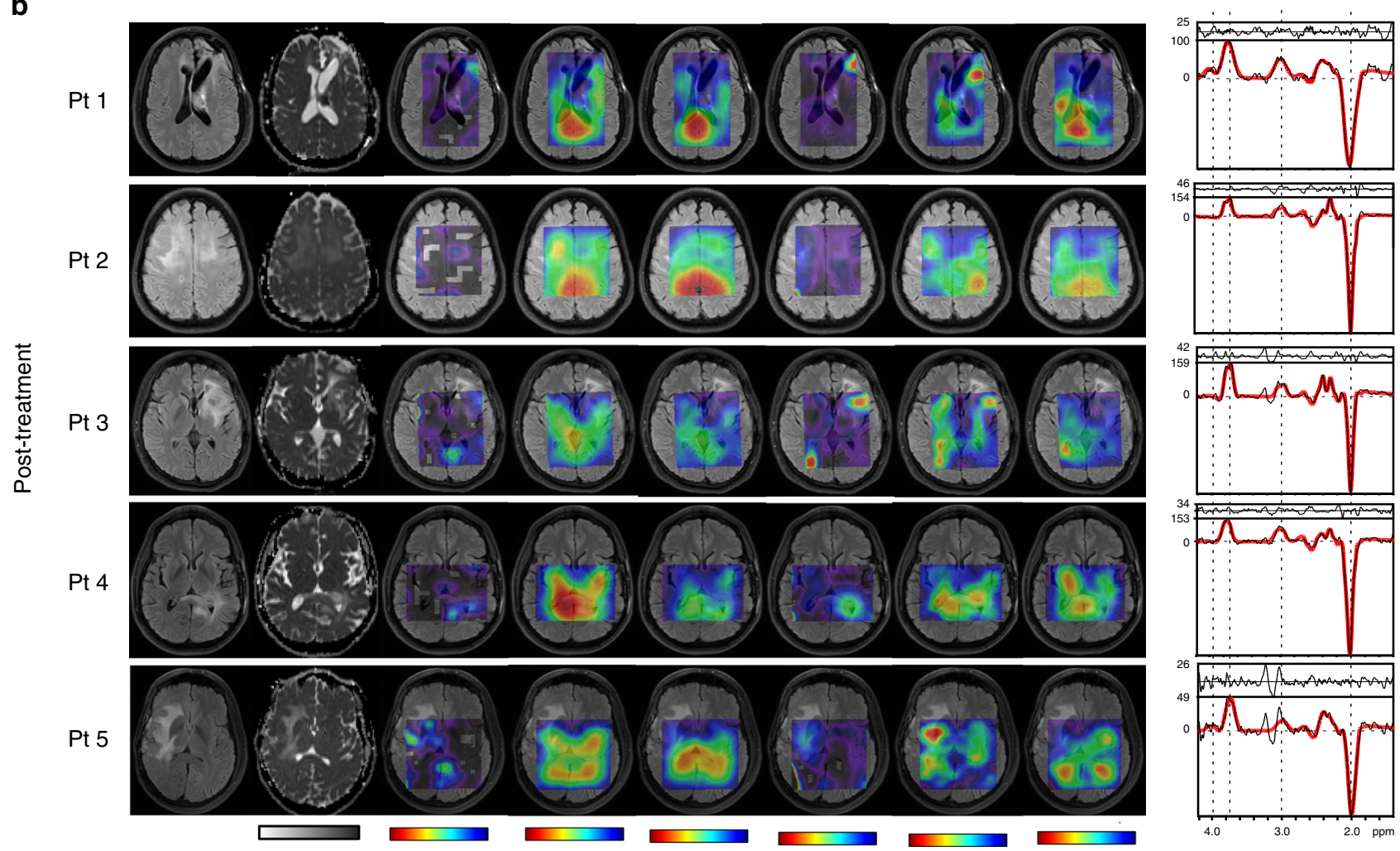

Fig. 1 Longitudinal imaging in mutant IDH1 glioma patients treated with IDH305 inhibitor. a Baseline anatomical, structural and metabolic maps before treatment. b Follow-up anatomical, structural, and metabolic maps after 1 week of IDH305 treatment. Metabolic maps are converted to mM by reference to healthy creatine levels. The color bars ranges are 0-3 mM (2HG), 0-20 mM (Glx), 0-1 mM (GSH), 0-16 mM (Lac), 0-3 mM (tCho), 0-20 mM (NAA), and $0-4 \mu \mathrm{m}^{2} / \mathrm{ms}(A D C)$. On the right, examples of $2 \mathrm{HG}$ edited spectra (black trace) and the LCModel fits (red trace) are shown 

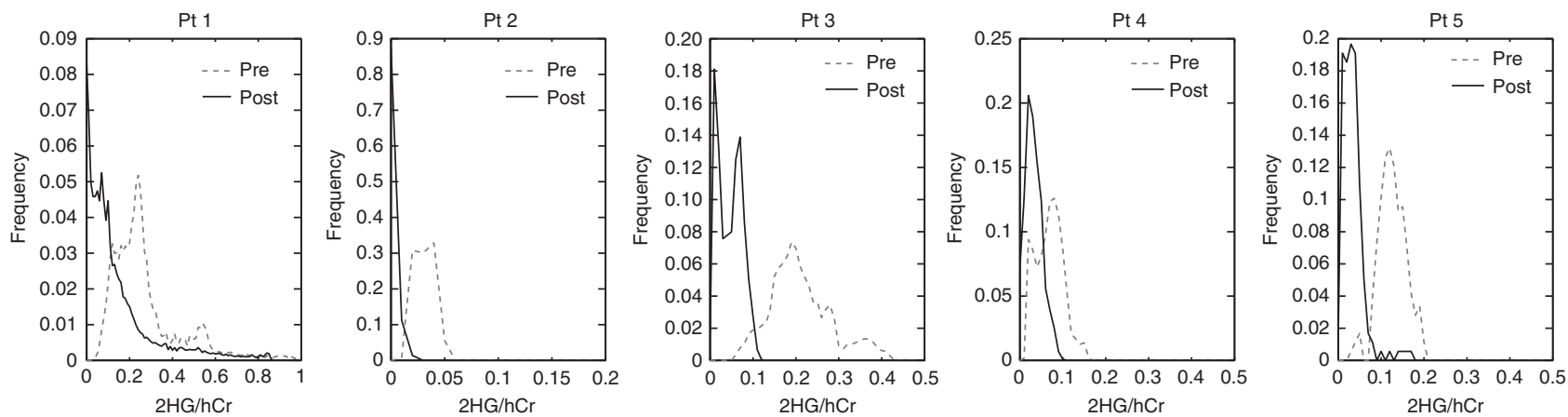

Fig. 2 Histograms of 2HG levels. The distribution of patient 2HG levels within the tumor ROI are shown before (dashed line) and after 1 week (continuous line) of treatment with the mutant IDH1 inhibitor IDH305

coupled with $2 \mathrm{HG}$ production, such as glutamate, glutamine, glutathione, and lactate. We investigated the relation between the change in $2 \mathrm{HG}$ levels and other metabolites, and the correlation with other imaging parameters such as tumor volumetrics, and apparent diffusion coefficient (ADC). The scope of this analysis was to uncover the mechanisms that explain the action of the mutant IDH1 inhibitors in glioma patients, and determine a comprehensive panel of imaging biomarkers that can be used to objectively assess the response to mutant $I D H 1$ inhibition. By combining genetics, pharmacology, and radiology, we demonstrate a comprehensive strategy, which we name radiopharmacodynamics, that can be used for precision and personalized medicine.

\section{Results}

Histological grading and molecular subtyping of patients. Eight patients were enrolled in Phase I clinical trial of IDH305 at our institution. Distribution of patients based on the 2016 WHO classification $^{3}$ was two grade II, five grade III, and one grade IV. Table 1 lists the demographics, histological grading/typing and the molecular subtyping, FLAIR and 2HG levels at baseline for all the patients.

Longitudinal metabolic imaging of patients. Longitudinal imaging data was available from five patients (\#1-5), all receiving $500 \mathrm{mg}$ of IDH305 orally BID. One patient (\#6) had only baseline imaging and did not complete the post treatment scan, while in two other patients (\#7 with grade II diffuse astrocytoma, and \#8 with grade III anaplastic astrocytoma) the 2HG levels at pretreatment imaging scan were not detectable by MRSI. These two patients (\#7 and \#8) had the smallest FLAIR volumes among all patients, 3.8 and $7.8 \mathrm{~cm}^{3}$, respectively. The $2 \mathrm{HG}$ imaging results from these three patients (\#6-8) are shown in Supplementary Figure 1. A recent study has found that MRS detection of $2 \mathrm{HG}$ is significantly reduced in patients with FLAIR volumes smaller than $8 \mathrm{~cm}^{3}$. The patient with $7.8 \mathrm{~cm}^{3}$ FLAIR volume had the shortest time interval ( 2 weeks) between the surgery and the baseline scan, with visible blood products in the surgical cavity. The tumor location in both patients was in a brain region which yielded good quality MRSI (SNR $>5$, FWHM $<12 \mathrm{~Hz}$ ).

Figure 1 shows longitudinal 3D imaging of $2 \mathrm{HG}$ and other brain metabolites in glioma patients (\#1-5) before and after 1 week of mutant $I D H 1$ inhibitor treatment with the experimental drug IDH305. Anatomical FLAIR and structural diffusion MR imaging acquired at the same time points are also presented. In the five patients with longitudinal $2 \mathrm{HG}$ imaging, the highest levels of $2 \mathrm{HG}$ are obtained in the region of FLAIR abnormality at the baseline scan. In some cases, high $2 \mathrm{HG}$ levels are noticed further away from the FLAIR region. However, by imposing a threshold

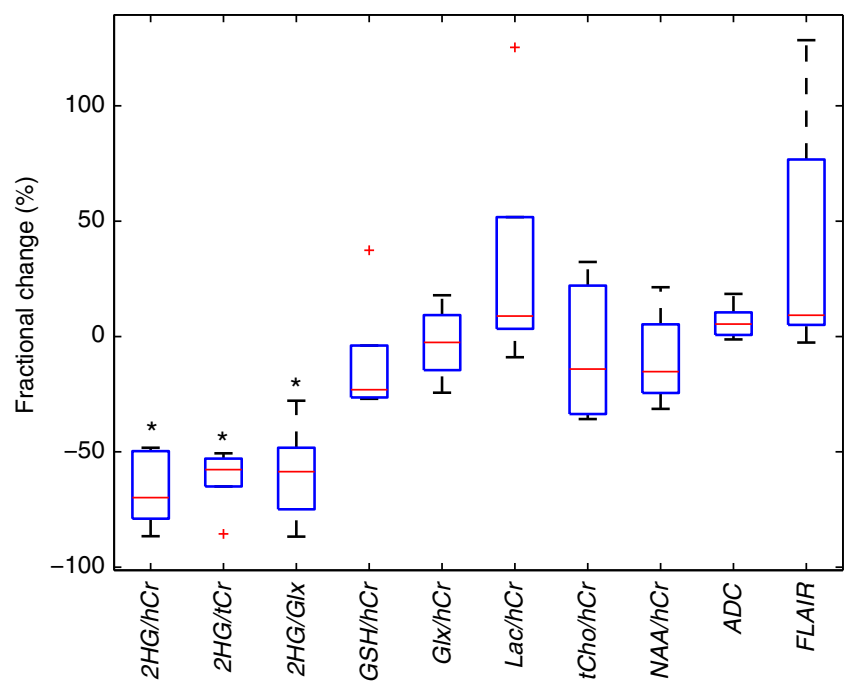

Fig. 3 Boxplot of the fractional changes for the mean values averaged over the tumor ROI. Box size represents the first and third quartiles, and horizontal red line indicates the median. Statistical significant changes are indicated by the ${ }^{*}$ symbol $(P<0.05)$

on the goodness of fit (Cramer Rao Lower Bounds, CRLB $<25 \%$ ) for 2HG signal, we obtain the mask with reliable 2HG quantification, as shown in Supplementary Figure 2 where the MRSI voxels remote from the tumor are eliminated.

Tumor 2HG levels decrease during mutant IDH1 inhibition. The distribution of $2 \mathrm{HG}$ values over the tumor volume and how this changed with the treatment was compared using histogram analysis. Histograms of tumor $2 \mathrm{HG}$ levels before and after treatment are shown in Fig. 2. The pretreatment histogram distributions are wider with a right tail toward higher values, while the left tail does not include values that are close to zero. The posttreatment histograms are narrower, shifted toward the left, and are asymmetric having the largest contribution toward zero.

The fractional change of mean values averaged over the entire tumor region of interest (ROI) is shown in Fig. 3 for 2HG, other brain metabolites, metabolic ratios, the apparent diffusion coefficient (ADC), and the ROI volume. After 1 week of treatment only the levels of $2 \mathrm{HG}$ show statistically significant $(P<0.05)$ changes with $70 \%$ reduction of the $2 \mathrm{HG} / \mathrm{hCr}$ ratio (2 $\mathrm{HG}$ relative to healthy creatine), $57 \%$ reduction of the $2 \mathrm{HG} / \mathrm{tCr}$ ratio (2HG relative to tumor creatine), and $58 \%$ reduction of the $2 \mathrm{HG} / \mathrm{Glx}$ ratio (2HG relative to glutamine + glutamate). There was a trend for an increase in lactate levels (9\%), increase in ADC 
Table 2 Quantification of metabolites and imaging parameters

\begin{tabular}{llllllllll} 
& $\mathbf{2 H G} / \mathbf{h C r}$ & $\mathbf{2 H G} / \mathbf{t C r}$ & $\mathbf{2 H G} / \mathbf{G l x}$ & $\mathbf{G S H} / \mathbf{h C r}$ & $\mathbf{G l x} / \mathbf{h C r}$ & $\mathbf{L a c} / \mathbf{h C r}$ & $\mathbf{t C h o / h C r}$ & $\mathbf{N A A} / \mathbf{h C r}$ & $\begin{array}{l}\text { ADC } \\
\mathbf{m s})\end{array}$ \\
\hline Pre (median) & 0.131 & 0.185 & 0.128 & 0.045 & 1.001 & 0.627 & 0.244 & 0.617 & 1.206 \\
Pre (95\% CI) & 0.091 & 0.163 & 0.074 & 0.008 & 0.133 & 0.433 & 0.065 & 0.130 & 0.095 \\
Post (median) & 0.057 & 0.102 & 0.064 & 0.039 & 0.974 & 0.760 & 0.226 & 0.626 & 1.312 \\
Post (95\% CI) & 0.043 & 0.078 & 0.057 & 0.006 & 0.217 & 0.314 & 0.087 & 0.220 & 0.096 \\
P-value & 0.019 & 0.017 & 0.035 & 0.263 & 0.807 & 0.308 & 0.706 & 0.545 & 0.132 \\
\hline
\end{tabular}

Median values, 95\% Confidence Intervals (CI), and the P-value for the pre-treatment and post-treatment imaging biomarkers. Inhibitors of the mutant isocitrate dehydrogenase 1 (IDH7) entered recently in clinical trials for the treatment of gliomas. Here, the authors apply a MRS imaging method for $2 \mathrm{HG}$ detection and assessement of the pharmacodynamic effects of the mutant IDH1 inhibitor (IDH305) in in clinical trials for the treatment of
eight mutant $I D H 1$ glioma patients.

(5\%), and increase in FLAIR volume (9\%). The median and confidence intervals of pre and posttreatment mean values across five patients are listed in Table 2.

In three patients, we had a third follow-up MR scan performed at 1 month after the start of IDH305 therapy. The 2HG levels $(2 \mathrm{HG} / \mathrm{hCr})$ at 1 month (Median $=0.06, \mathrm{CI}=0.07)$ and at 1 week (Median $=0.05, C I=0.03$ ) are similarly decreased, compared to the baseline (Median $=0.21, \mathrm{CI}=0.1$ ). There is increased variability at 1 month because the $2 \mathrm{HG}$ levels drop toward the detectability threshold for MRS, hence the precision of our estimates decreases. We have included in Supplementary Figure 3 the $2 \mathrm{HG}$ imaging data for the third visit and in Supplementary Figure 4 the plot of $2 \mathrm{HG}$ values for all three time points.

Metabolic reprogramming of mutant IDH1 glioma. Next, we investigated whether there is a relationship between the change in $2 \mathrm{HG}$ levels and the metabolites that are coupled with $2 \mathrm{HG}$ production. In particular, glutathione, glutamine, and glutamate are a part of the metabolic reactions that are closely connected with the mutant IDH1 pathway. These metabolites have important cellular roles, GSH is the major scavenger of free radical species required to maintain the redox balance, and glutamine is an anaplerotic source of carbon and nitrogen in the brain. In addition to these, lactate is a metabolite of particular significance in cancer, related to the Warburg effect, and is implicated in many cancer mechanisms.

The increase in lactate levels shown in Fig. 3 may be an important observation associated with the inhibition of mutant $I D H 1$. It has been reported that the activity of lactate dehydrogenase A (LDH-A) is downregulated in mutant IDH1 glioma cells ${ }^{44}$, which is opposite to the typical upregulation of LDH-A in most other cancers.

The mutant $I D H 1$ couples the reduction of aKG to $2 \mathrm{HG}$ with the oxidation of $\mathrm{NADPH}$ to $\mathrm{NADP}^{+}$. It has been shown that wild-type $I D H 1$ is the most important source of NADPH in the brain, and the mutant IDH1 enzyme consumes more NADPH than produced by the wild-type IDH1 enzyme ${ }^{45}$. In turn, $\mathrm{NADPH}$ is used to regenerate the reduced glutathione, and a decrease in NADPH in mutant IDH1 glioma may lower the GSH levels. Conversely, an increase in NADPH due to inhibition of mutant IDH1 may result in an increase in GSH levels. A linear model fit in Fig. 4a shows a trend for inverse correlation between fractional changes of $2 \mathrm{HG}$ and GSH levels.

Glutamine is an important source for tricarboxylic acid cycle anaplerosis in cancer cells ${ }^{46}$, and in the case of mutant IDH1 glioma cells, glutamine becomes the major source for $2 \mathrm{HG}$ synthesis via a two-step process, involving glutamate and aKG as intermediaries ${ }^{4}$. Glutamate and glutamine levels have been found to be lower in mutant $I D H 1$ compared to wild-type $I D H 1$ glioma patients, as measured by the mass spectrometry in tumor biopsies ${ }^{47}$. Previously, we found that Glx signal (glutamate + glutamine) by in vivo MRSI is decreased in tumor, compared to contralateral normal appearing white matter in mutant IDH1 patients. Inhibition of mutant IDH1 activity might limit the conversion of glutamine into 2HG. Our results from Fig. 4b indicate a trend for inverse correlation between the fractional changes of $2 \mathrm{HG}$ and Glx, however this correlation is weaker than in the case of GSH.

Correlation of metabolic profile and cellular density. Previous studies ${ }^{48}$ have found that $2 \mathrm{HG}$ levels correlate with the cellular density of mutant IDH1 glioma tumors. In this study, we sought to investigate whether the dynamic changes in $2 \mathrm{HG}$ levels would correlate with the dynamic changes in cell density during treatment with mutant IDH1 inhibitors. Since longitudinal biopsies were not available, we correlated the changes in $2 \mathrm{HG}$ with the changes in an imaging parameter that is a surrogate biomarker for cell density. The apparent diffusion coefficient is an imaging biomarker that probes tissue microstructure and cell density ${ }^{49,50}$. $\mathrm{ADC}$ has been shown to increase in tumors that respond to the treatment ${ }^{51,52}$. The results of a linear model fit in Fig. $4 \mathrm{c}$ shows that there is a significant inverse correlation between the changes in the $2 \mathrm{HG} / \mathrm{Glx}$ ratio and the changes in ADC. In the case of mutant IDH1 inhibition, the simultaneous decrease of the 2HG/ Glx ratio and the increase in ADC might be interpreted as a sign of objective treatment response in glioma patients.

\section{Discussion}

Mutations of IDH1 offer the opportunity to develop an effective treatment against gliomas. The efforts to translate mutant IDH1targeted treatments are significantly enhanced by the existence of the $2 \mathrm{HG}$ biomarker that can be measured to assess the objective response rates. However, serial biopsies for pharmacodynamic assessments in glioma patients pose the risks of neurological injury or infection, which in the setting of clinical trials might not outweigh the clinical benefits. Hence, imaging ${ }^{53}$ and "liquid biopsies" 54 (blood, urine, and cerebrospinal fluid) remain the only options, at present, to objectively monitor the treatments over time in glioma trials. $2 \mathrm{HG}$ is the most informative biomarker on the pharmacodynamics of mutant IDH1 inhibitors. The intratumoral $2 \mathrm{HG}$ levels probe directly the in vivo activity of mutant IDH1 enzyme, while the peripheral 2HG levels are modulated in addition by other factors, such as the BBB. In particular, 3D imaging is well-suited to serially monitor the treatment because it can investigate the entire tumor volume and healthy brain, and it is not affected by sampling bias of single-point measurements. Hence, precision medicine-based clinical trials become feasible in glioma patients, by employing quantitative imaging for pharmacodynamic biomarker assessment in longitudinal studies.

The molecular mechanisms of the mutant IDH1 glioma are not fully understood, and the implications of mutant $I D H 1$ inhibition are still a matter of debate. Earlier, preclinical data demonstrated $^{15}$ that the mutant $I D H 1$ inhibition might be able to revert 
a

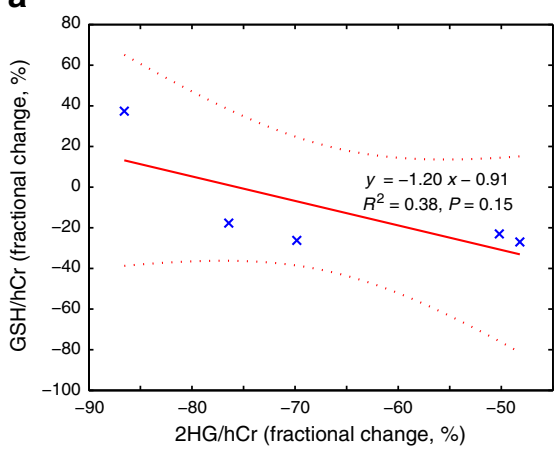

b

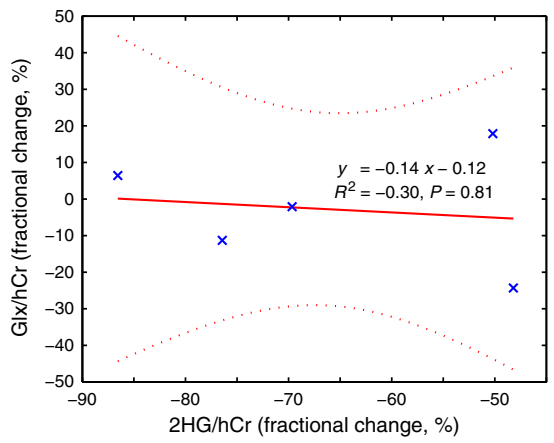

C

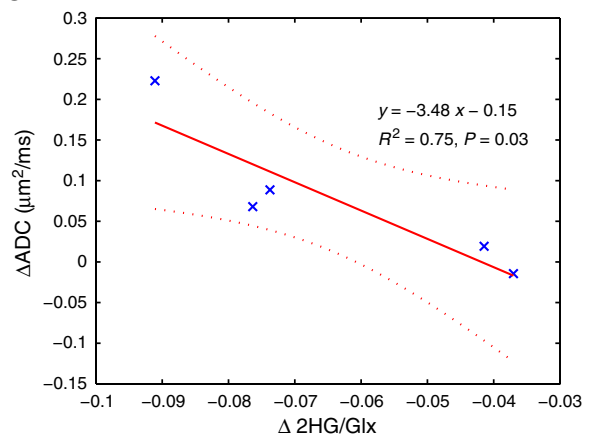

Fig. 4 Relation between the treatment induced changes of $2 \mathrm{HG}$ and other metabolic and imaging biomarkers. Linear model fitting has been employed to investigate the relationship between the changes: $\mathbf{a} 2 \mathrm{HG}$ and $\mathrm{GSH} ; \mathbf{b} 2 \mathrm{HG}$ and $\mathrm{Glx}$; $\mathbf{c}$ ratio of $2 \mathrm{HG} / \mathrm{Glx}$ and $\mathrm{ADC}$. A significant inverse correlation was found between changes of $2 \mathrm{HG} / \mathrm{Glx}$ ratio and changes of ADC after 1 week of treatment with IDH305

the histone hypermethylation, but not DNA hypermethylation, induce cell differentiation, and delay tumor growth. Recently, preclinical studies using multiple patient-derived glioma models have failed to show an effect on tumor growth by mutant IDH1 inhibitors ${ }^{18}$. More importantly, the clinical data show that mutant IDH1 patients have significantly longer survival compared to wild-type $I D H 1$ patients ${ }^{2}$, implying some benefit in harboring the IDH1 mutations either by a more indolent progression or intrinsic vulnerabilities that cause better response to existing therapies $^{14}$.

To unequivocally answer the conundrum regarding the implications of mutant IDH1 inhibition, it is paramount to probe the target modulation in patients and exclude the lack of in vivo drug activity. Our results demonstrate that IDH305 modulates target activity and is effective in the inhibition of mutant $I D H 1$, lowering $2 \mathrm{HG}$ levels by $70 \%$ after 1 week of treatment. In addition, there are other metabolic effects of mutant $I D H 1$ inhibition, as suggested by a trend toward an inverse correlation between changes of $2 \mathrm{HG}$ and glutathione or glutamine + glutamate, respectively. Opposite changes of $2 \mathrm{HG}$ and Glx levels in mutant IDH1 glioma (i.e., high $2 \mathrm{HG}$ and low Glx tumor levels) may make the $2 \mathrm{HG} / \mathrm{Glx}$ ratio a metabolic biomarker that has an increased range to detect mutant $I D H 1$ tumor tissue. Although similar to the Cho/NAA ratio (i.e., high Cho and low NAA tumor levels) in wild-type gliomas, the $2 \mathrm{HG} / \mathrm{Glx}$ ratio may be more specific to detect the targeted treatment response against mutant IDH1 gliomas. Changes in glutathione levels are particularly relevant in connection with tumor susceptibility to radiotherapy. The biological effects of radiotherapy are amplified by free radical oxygen species, which in turn are neutralized by glutathione. Hence, it has been hypothesized that mutant IDH1 patients having lower $\mathrm{NADPH}$, and consequently lower GSH levels, would be more radiosensitive $e^{45,55}$. On the other hand, an increase in GSH due to $\mathrm{mIDH} 1$ inhibition would diminish the radiobiological effects, and may argue against the simultaneous administration of mutant IDH1 inhibitors and radiotherapy ${ }^{56}$. The correlation between changes of Glx and $2 \mathrm{HG}$ is weaker than the one between the changes of GSH and $2 \mathrm{HG}$, which may be explained by the fact that glutamine is used in many metabolic pathways, including being a precursor for the synthesis of GSH. The increase in lactate levels in post-treatment scans could indicate that downregulation of the LDH-A activity is reversible through mutant IDH1 inhibition. Lactate is thought to play many roles in the pathophysiology of cancer, creating an acidic environment that favors cell migration, immune escape, and epigenetic alterations ${ }^{57}$. On the other hand, we observed a significant correlation between decreasing levels of $2 \mathrm{HG} / \mathrm{Glx}$ and increasing ADC values. An increase in ADC is associated with a decrease in cellular density, and is regarded as a positive outcome in cancer treatment. Thus, raising lactate levels and increasing ADC values by mutant $I D H 1$ inhibition poses a puzzling scenario.

In some patients, we noted an increase of the FLAIR volume which raises the question whether these patients experienced tumor progression during the treatment with IDH305. A related question is whether the quantification of $2 \mathrm{HG}$ levels is affected by the change in FLAIR volumes. In order to avoid the possible confounding effect due edema and changes in water content in tumor, we quantified $2 \mathrm{HG}$ and the other tumor metabolites relative to the levels of creatine in the normal appearing (healthy) brain which is not affected by IDH305, and where no change in FLAIR is noticed. Tumor $2 \mathrm{HG}$ levels also decrease when quantified, relative to healthy creatine, which indicate a true $2 \mathrm{HG}$ decrease. A true $2 \mathrm{HG}$ decrease and the decrease of total choline would reject the tumor progression and would be consistent with pseudo-progression radiographic changes. In addition, no worsening of clinical symptoms, such as progressive headaches were reported by the patients during the course of the study.

Metabolic maps of the mutant IDH glioma patients show that levels of glutamate/glutamine (Glx) and glutathione (GSH) are decreased in the tumor regions compared to the contralateral regions, while lactate is increased in the tumor. However, while $2 \mathrm{HG}$ and lactate are focally increased in the tumor, because of lack of background signal in the surrounding tissue, the maps of Glx and GSH show smaller contrast between the tumor and the healthy tissue due to larger background signal of the normal brain. In addition, the longitudinal changes of Glx could be confounded by different fate of glutamate and glutamine in tumor metabolism. Methods ${ }^{58-61}$ that can separate better the contributions of Glu and Gln in MR spectroscopy might be able to differentiate better whether the inhibition of $2 \mathrm{HG}$ in patients is coupled with biochemical pathways of Gln or Glu.

Several limitations exist in our study, the most important being the low number of patients. Phase I clinical trials focused on the safety/toxicity/feasibility are performed on a small cohort of patients, and our imaging data is inherently limited by the design of the Phase I study. In addition, recurrent glioma patients enrolled in the Phase I trial are particularly challenging for $2 \mathrm{HG}$ detection, since these patients have a history of multiple prior therapies, including surgical resections. Existing data ${ }^{27,28}$ indicate that current chemoradiation therapies are also effective in reducing $2 \mathrm{HG}$ levels. Hence, it is expected that the baseline $2 \mathrm{HG}$ levels in patients from the Phase I study would be lower than in newly diagnosed patients. In our study, we were not able to detect $2 \mathrm{HG}$ levels at baseline in $25 \%(2 / 8)$ of the patients, which was related to small residual tumor postsurgery. With our MRSI protocol, we can reliably detect $2 \mathrm{HG}$ concentrations above $1 \mathrm{mM}^{62}$, though 
lower concentrations $(0.1-1 \mathrm{mM})$ are possible for data of very good spectral quality (linewidth $<6 \mathrm{~Hz}$ ). Assuming $2 \mathrm{HG}$ concentration of $1 \mathrm{mM}$, tumors that have a volume of $1.9 \mathrm{~cm}^{3}$ or larger can be detected with our current protocol, but smaller tumors may be detected if they produce higher $2 \mathrm{HG}$ levels, or by more data averaging and longer acquisition times. These limitations due to imaging protocol mandates, further the efforts to improve the sensitivity of MRS-based 2HG detection. Another limitation is related to the fact that we do not yet have survival data to correlate with imaging parameters. Hence, we are not able, at this moment, to make any analysis regarding the predictive value of the objective response assessment based on $2 \mathrm{HG}$ levels, nor to infer any conclusion regarding the patient benefit. Despite these limitations, our preliminary results from this Phase I study shed light on the metabolic networks that may be impacted in patients by the inhibition of mutant $I D H 1$, and potentially guide the future development. Although interesting, our imaging results need to be replicated in a larger cohort of patients.

Our radiopharmacodynamics study demonstrates the feasibility of image-based 2HG pharmacodynamic serial assessments in patients treated with mutant $I D H 1$ inhibitors. Since no animal model entirely recapitulates the human disease, there is no better substitute for human studies to test these drugs. A precision medicine approach is easy to perform by non-invasive longitudinal imaging in glioma patients where serial biopsies have high risks. Although, we found correlation between $2 \mathrm{HG}$ levels and widely used imaging parameters, such as ADC, the $2 \mathrm{HG}$ measurements are indispensable to probe the target modulation, mechanisms of action, metabolic reprogramming, and further could be used to study the dose-response activity of mutant IDHI inhibitors.

2HG measurements have the highest specificity for mutant IDH1 mutations, and may distinguish true/pseudo-response/ progression in the case of standard chemoradiation. However, we would like to caution that in the case of targeted mutant IDHI inhibition the interpretation and role of $2 \mathrm{HG}$ imaging needs to be carefully evaluated and understood. For example, mutant IDH1 inhibition could efficiently reduce the $2 \mathrm{HG}$ levels, but this may not equate with tumor cell death. This is a situation analogous to that of anti-angiogenic therapy that reduces the contrast enhancement making tumors almost invisible on T1-weighted MRI, although gliomas can persist and progress behind a restored $\mathrm{BBB}^{40}$. Thus, decreased $2 \mathrm{HG}$ in the setting of mutant IDH1 inhibition could be similar to reduced BBB leakiness of Gd-based contrast agents in the setting of anti-angiogenic therapy, and the pseudo-response by $2 \mathrm{HG}$ imaging might not be excluded. Nevertheless, the possibility of a $2 \mathrm{HG}$ imaging pseudo-response is likely to happen only in the case of mutant IDH1 inhibitors used as monotherapy. In the case of standard chemoradiation, immunotherapy, or combination therapies, a decrease in $2 \mathrm{HG}$ is likely to reflect a decrease in tumor cell numbers. On the other hand, an increase of $2 \mathrm{HG}$ can rule out pseudo-progression and rule in true-progression. It is interesting to point that our data shows a tendency for larger FLAIR volumes after mutant IDH1 inhibition, which may suggest either pseudo-progression or truedisease progression. The simultaneous increase of ADC with decrease of $2 \mathrm{HG}$ supports, in this case, the pseudo-progression interpretation of imaging findings. $2 \mathrm{HG}$ is likely the earliest biomarker of response in the case of mutant $I D H 1$ inhibition, and a disconnect between $2 \mathrm{HG}$ and conventional anatomical imaging is expected. To account for such a complex scenario, a larger panel of imaging biomarkers should be used in addition to $2 \mathrm{HG}$, including other metabolites such as lactate, Glx, choline, NAA, and structural parameters such as ADC.

In summary, we demonstrate that $2 \mathrm{HG}$ imaging can inform the phase I clinical trial on the biological effects of targeted therapies against mutant IDH1 gliomas, and potentially it could be used to guide future trial designs. An early assessment of treatment is important to avoid the possible negative side effects, and steer the course for maximizing patient benefit and quality of life. We expect that our methodology will help efforts of cancer scientists for further rational drug development.

\section{Materials and methods}

Patient population. Patients were enrolled in the imaging study, according to the inclusion/exclusion criteria of the Phase I clinical trial (ClinicalTrials.gov identifier NCT02381886). Briefly, to be included the patients had to have confirmed IDH1 R132 mutant glioma for whom no curative therapy is available and who have evidence of disease progression following a standard therapy. Patients were excluded if they had less than 2 weeks from the last dose of systemic antineoplastic therapy, 6 weeks from bevacizumab, 3 months from radiotherapy, and less than 2 weeks from major surgery. In seven patients the IDH305 was administered at a dose of $550 \mathrm{mg}$ orally twice a day (BID), while in one patient the dose was $900 \mathrm{mg}$ orally BID. Imaging was performed at baseline 1-7 days prior to the start of IDH305, and at follow-up after 7 days of IDH305. The choice of the follow-up time point at 1 week was determined based on the following reasons: (1) a rapid inhibition of $\mathrm{mIDH} 1$ by IDH305 with a large effect size on 2HG levels and (2) unknown toxicity that may limit the duration of IDH305 administration.

IDH1 mutations. IDH1-mutational status was first tested by immunohistochemistry (IHC) analysis using an anti-human $\mathrm{R} 132 \mathrm{H}$ antibody (DIANOVA) ${ }^{63}$ and subsequently confirmed by genetic sequencing (SNaPshot) ${ }^{64,65}$. Other molecular markers such as $1 \mathrm{p} / 19 \mathrm{q}$ codeletion ${ }^{66}$ and MGMT promoter methylation ${ }^{67}$ were tested using fluorescence in situ hybridization (FISH) and polymerase chain reaction (PCR), respectively ${ }^{64}$, while TP53 mutation was confirmed also by SNaPshot ${ }^{64,65}$

Imaging protocol. MRI and MRSI data were acquired on a clinical 3T MR scanner (Tim Trio, Siemens, Erlangen) with a 32-channel head coil. A recently developed spectral-editing 3D MRSI sequence was used for metabolic imaging as following. Two spectral editing 3D MRSI acquisitions were performed (1) for $2 \mathrm{HG}$ detection $^{27}$ with MEGA editing pulses applied at $1.9 \mathrm{ppm}(\mathrm{ON})$ and $7.5 \mathrm{ppm}(\mathrm{OFF})$ and (2) for GSH detection ${ }^{68}$ with MEGA editing pulses applied at $4.57 \mathrm{ppm}(\mathrm{ON})$ and $-1 \mathrm{ppm}$. Other acquisition parameters of 3D MRSI were: MEGA-LASER spectral editing, spiral k-space readout, $\mathrm{TR}=1600 \mathrm{~ms}, \mathrm{TE}=68 \mathrm{~ms}, \mathrm{FOV}=200 \times 200 \times$ $200 \mathrm{~mm}^{3}$, VOI $=100 \times 80 \times 50 \mathrm{~mm}^{3}$, acquisition matrix $10 \times 10 \times 10$ which was zero filled to $16 \times 16 \times 16$ in the FFT reconstruction, yielding a digital voxel volume of $1.9 \mathrm{~cm}^{3}$, acquisition time 9:55 min:s. Second order B0 shimming was performed based on B0 fieldmaps obtained with a double echo gradient sequence. Water suppression was performed with WET scheme ${ }^{69}$. Anatomical and structural MRI was performed using FLAIR $($ TR/TI/TE $=10000 / 2500 / 70 \mathrm{~ms}, \mathrm{FOV}=220 \times 186 \times$ $138 \mathrm{~mm}^{3}$, matrix $\left.=256 \times 192 \times 23\right)$ and diffusion tensor imaging $(\mathrm{TR} / \mathrm{TE}=7980 /$ $84 \mathrm{~ms}, b$-values $=0 / 700 \mathrm{~s} / \mathrm{mm}^{2}$, gradient directions $=12, \mathrm{FOV}=236 \times 236 \times 118$ $\mathrm{mm}^{3}$, matrix $=128 \times 128 \times 64$ ). To reproduce the positioning across visits for each patient, the online automatic slice positioning (AAscout ${ }^{70}$ ) was used during acquisition of all imaging data.

Image analysis. Difference and OFF spectra from the 3D MRSI acquisition were fitted with LCModel ${ }^{71}$ software using the corresponding simulated basis sets for the MEGA-LASER sequence. Difference spectra were used to quantify $2 \mathrm{HG}$, Glx (glutamate and glutamine), and glutathione (GSH). The OFF spectra were used to quantify the total choline (tCho), creatine (Cr), lactate (Lac), and N-acetylaspartate (NAA). Linewidth less than $0.1 \mathrm{ppm}(12 \mathrm{~Hz})$ and Cramer-Rao lower bound (CRLB) less than $25 \%$ was considered for goodness of fit of $2 \mathrm{HG}$, Glx, and GSH in difference spectra. CRLB less than $20 \%$ was considered for the goodness of fit of tCho, Cr, Lac, and NAA in OFF spectra. The 3D metabolic maps were reconstructed using a combination of MINC (Montreal Neurological Institute), FSL (FMRIB Software Library, Oxford, UK), and MATLAB (Mathworks, Natick, MA) software tools. For all metabolites, relative maps were calculated by dividing the metabolite to healthy creatine ( $\mathrm{hCr}$ ) averaged in a ROI situated in contralateral normal appearing white matter. Healthy creatine levels were assumed to be $8 \mathrm{mM}$, and this was used to convert the units of the relative metabolic maps to $\mathrm{mM}$ concentration units. For $2 \mathrm{HG}$, relative maps were calculated also relative to the creatine $(2 \mathrm{HG} / \mathrm{tCr})$ and $\mathrm{Glx}$ in the tumor. Tumor ROIs were manually outlined around the hyperintense areas in the FLAIR images. Healthy ROIs were drawn in contralateral hemisphere in a region that appeared normal on FLAIR images. Using the upper threshold for CRLB $(<25 \%)$, we obtained the ROI masks that select the voxels with reliable $2 \mathrm{HG}$ quantification. The ROIs for tumor, normal appearing white matter, and the $2 \mathrm{HG}$ goodness of fit mask $(\mathrm{CRLB}<25 \%)$ are shown in Supplementary Figure 1.

Statistical analysis. Differences in imaging biomarkers between post- and pre treatment scans were deemed significant at a level of $P<0.05$ using two-sided 
paired Student's $t$-test. The data normality was verified with Kolmogorov-Smirnov test. Correlations between $2 \mathrm{HG}$ and other imaging biomarkers were calculated using Pearson product-moment correlation coefficient. Linear model fit was employed to establish the relation between 2HG and other biomarkers. All statistical analysis was performed with the statistical package of MATLAB.

Data and materials availability. The datasets generated during and/or analyzed during the current study are available from the corresponding author on reasonable request.

Received: 8 July 2017 Accepted: 21 March 2018

Published online: 16 April 2018

\section{References}

1. Parsons, D. W. et al. An integrated genomic analysis of human glioblastoma multiforme. Science 321, 1807-1812 (2008)

2. Yan, H. et al. IDH1 and IDH2 mutations in gliomas. New Engl. J. Med. 360, 765-773 (2009).

3. Louis, D. N. et al. The2016 World Health Organization classification of tumors of the central nervous system: a summary. Acta Neuropathol. 131, 803-820 (2016).

4. Dang, L. et al. Cancer-associated IDH1 mutations produce 2-hydroxyglutarate. Nature 462, 739-744 (2009).

5. $\mathrm{Xu}, \mathrm{W}$. et al. Oncometabolite 2-hydroxyglutarate is a competitive inhibitor of alpha-ketoglutarate dependent dioxygenases. Cancer Cell 19, 17-30 (2011).

6. Noushmehr, H. et al. Identification of a CpG island methylator phenotype that defines a distinct subgroup of glioma. Cancer Cell 17, 510-522 (2010).

7. Turcan, S. et al. IDH1 mutation is sufficient to establish the glioma hypermethylator phenotype. Nature 483, 479-483 (2012).

8. Chowdhury, R. et al. The oncometabolite 2-hydroxyglutarate inhibits histone lysine demethylases. Embo. Rep. 12, 463-469 (2011).

9. Lu, C. et al. IDH mutation impairs histone demethylation and results in a block to cell differentiation. Nature 483, 474-478 (2012).

10. Koivunen, P. et al. Transformation by the (R)-enantiomer of 2hydroxyglutarate linked to EGLN activation. Nature 483, 484-488 (2012).

11. Beiko, J. et al. IDH1 mutant malignant astrocytomas are more amenable to surgical resection and have a survival benefit associated with maximal surgical resection. Neuro. Oncol. 16, 81-91 (2014).

12. Cairncross, J. G. et al. Benefit from procarbazine, lomustine, and vincristine in oligodendroglial tumors is associated with mutation of IDH. J. Clin. Oncol. 32, 783-790 (2014).

13. Kizilbash, S. H. et al. The impact of concurrent temozolomide with adjuvant radiation and IDH mutation status among patients with anaplastic astrocytoma. J. Neurooncol. 120, 85-93 (2014).

14. Tran, A. N. et al. Increased sensitivity to radiochemotherapy in IDHI mutant glioblastoma as demonstrated by serial quantitative MR volumetry. Neuro. Oncol. 16, 414-420 (2014).

15. Rohle, D. et al. An inhibitor of mutant $I D H 1$ delays growth and promotes differentiation of glioma cells. Science 340, 626-630 (2013).

16. Schumacher, T. et al. A vaccine targeting mutant $I D H 1$ induces antitumour immunity. Nature 512, 324-327 (2014).

17. Sulkowski, P. L. et al. 2-Hydroxyglutarate produced by neomorphic IDH mutations suppresses homologous recombination and induces PARP inhibitor sensitivity. Sci. Transl. Med. 9, eaal2463 (2017).

18. Tateishi, K. et al. Extreme vulnerability of IDH1 mutant cancers to NAD +depletion targetable signaling pathway mutations are associated with malignant phenotype in IDH-mutant gliomas. Cancer Cell 28, 773-784 (2015).

19. Reardon, D. A., Ballman, K. V., Buckner, J. C., Chang, S. M. \& Ellingson, B. M. Impact of imaging measurements on response assessment in glioblastoma clinical trials. Neuro. Oncol. 16, vii24-vii35 (2014).

20. Ellingson, B. M., Bendszus, M., Sorensen, A. G. \& Pope, W. B. Emerging techniques and technologies in brain tumor imaging. Neuro. Oncol. 16, vii12-vii23 (2014)

21. Sullivan, D. C. et al. Metrology standards for quantitative imaging biomarkers. Radiology 277, 813-825 (2015).

22. Upadhyay, V. A., Brunner, A. M. \& Fathi, A. T. Isocitrate dehydrogenase (IDH) inhibition as treatment of myeloid malignancies: progress and future directions. Pharmacol. Ther. 14, 30083-30089 (2017).

23. Choi, C. et al. 2-Hydroxyglutarate detection by magnetic resonance spectroscopy in subjects with IDH-mutated gliomas. Nat. Med. 18, 624-629 (2012).
24. Andronesi, O. C. et al. Detection of 2-hydroxyglutarate in IDH-mutated glioma patients by in vivo spectral-editing and 2D correlation magnetic resonance spectroscopy. Sci. Transl. Med. 4, 116ra114 (2012).

25. Emir, U. E. et al. Noninvasive quantification of 2-hydroxyglutarate in human gliomas with IDH1 and IDH2 mutations. Cancer Res. 76, 43-49 (2016).

26. Kalinina, J. et al. Detection of "oncometabolite" 2-hydroxyglutarate by magnetic resonance analysis as a biomarker of $I D H 1 / 2$ mutations in glioma. $J$. Mol. Med. (Berl.) 90, 1161-1171 (2012).

27. Andronesi, O. C. et al. Treatment response assessment in IDH-mutant glioma patients by non-invasive 3D functional spectroscopic mapping of 2hydroxyglutarate. Clin. Cancer Res. 22, 1632-1641 (2016).

28. Choi, C. et al. Prospective longitudinal analysis of 2-hydroxyglutarate magnetic resonance spectroscopy identifies broad clinical utility for the management of patients with IDH-mutant glioma. J. Clin. Oncol. 34, 4030-4039 (2016)

29. Nakamizo, S. et al. GC/MS-based metabolomic analysis of cerebrospinal fluid (CSF) from glioma patients. J Neurooncol. https://doi.org/10.1007/s11060013-1090-x (2013).

30. Lombardi, G. et al. Diagnostic value of plasma and urinary 2-hydroxyglutarate to identify patients with isocitrate dehydrogenase-mutated glioma. Oncologist 20, 562-567 (2015).

31. Capper, D. et al. 2-Hydroxyglutarate concentration in serum from patients with gliomas does not correlate with $I D H 1 / 2$ mutation status or tumor size. Int. J. Cancer 131, 766-768 (2012).

32. Fathi, A. T. et al. Elevation of urinary 2-hydroxyglutarate in IDH-mutant glioma. Oncologist 1, 2015-0342 (2016).

33. Kalinina, J. et al. Selective detection of the D-enantiomer of 2hydroxyglutarate in the CSF of glioma patients with mutated isocitrate dehydrogenase. Clin. Cancer Res.: Off. J. Am. Assoc. Cancer Res. 22, 6256-6265 (2016).

34. Carrillo, J. A. et al. Relationship between tumor enhancement, edema, IDHI mutational status, MGMT promoter methylation, and survival in glioblastoma. Am. J. Neuroradiol. 33, 1349-1355 (2012).

35. Lee, S. et al. Evaluation of the microenvironmental heterogeneity in highgrade gliomas with $I D H 1 / 2$ gene mutation using histogram analysis of diffusion-weighted imaging and dynamic-susceptibility contrast perfusion imaging. J. Neurooncol. 121, 141-150 (2014).

36. Tan, W. L. et al. Can diffusion tensor imaging noninvasively detect $I D H 1$ gene mutations in astrogliomas? a retrospective study of 112 cases. Am. J. Neuroradiol. 35, 920-927 (2014).

37. Kickingereder, P. et al. IDH mutation status is associated with a distinct hypoxia/angiogenesis transcriptome signature which is non-invasively predictable with rCBV imaging in human glioma. Sci. Rep. 5, 16238 (2015).

38. Patel, S. H. et al. T2-FLAIR mismatch, an imaging biomarker for IDH and $1 \mathrm{p} /$ 19q status in lower-grade gliomas: a TCGA/TCIA project. Clin. Cancer Res.: Off. J. Am. Assoc. Cancer Res. 23, 6078-6085 (2017).

39. Delfanti, R. L. et al. Imaging correlates for the 2016 update on WHO classification of grade II/III gliomas: implications for IDH, 1p/19q and ATRX status. J. Neurooncol. https://doi.org/10.1007/s11060-017-2613-7 (2017).

40. Brandsma, D., van den Bent, M. J., Shim, H., Holder, C. A. \& Olson, J. J. Pseudoprogression and pseudoresponse in the treatment of gliomas magnetic resonance spectroscopic imaging in the era of pseudoprogression and pseudoresponse in glioblastoma patient management. Curr. Opin. Neurol. 22, 633-638 (2009).

41. Wen, P. Y. et al. Updated response assessment criteria for high-grade gliomas: response assessment in neuro-oncology working group. J. Clin. Oncol. 28, 1963-1972 (2010)

42. van den Bent, M. J. et al. Response assessment in neuro-oncology (a report of the RANO group): assessment of outcome in trials of diffuse low-grade gliomas. Lancet Oncol. 12, 583-593 (2011).

43. Leu, K. et al. Perfusion and diffusion MRI signatures in histologic and genetic subtypes of WHO grade II-III diffuse gliomas. J. Neurooncol. 134, 177-188 (2017).

44. Chesnelong, C. et al. Lactate dehydrogenase A silencing in IDH mutant gliomas. Neuro. Oncol. 16, 686-695 (2014).

45. Bleeker, F. E. et al. The prognostic IDH1 (R132) mutation is associated with reduced NADP(+)-dependent IDH activity in glioblastoma. Acta Neuropathol. 119, 487-494 (2011).

46. Daye, D. \& Wellen, K. E. Metabolic reprogramming in cancer: unraveling the role of glutamine in tumorigenesis. Semin. Cell. Dev. Biol. 23, 362-369 (2012).

47. Ohka, F. et al. Quantitative metabolome analysis profiles activation of glutaminolysis in glioma with IDH1 mutation. Tumour Biol. 35, 5911-5920 (2014).

48. de la Fuente, M. I. et al. Integration of 2-hydroxyglutarate-proton magnetic resonance spectroscopy into clinical practice for disease monitoring in isocitrate dehydrogenase-mutant glioma. Neuro. Oncol. 18, 283-290 (2016). 
49. Kalpathy-Cramer, J., Gerstner, E. R., Emblem, K. E., Andronesi, O. C. \& Rosen, B. Advanced Magnetic Resonance Imaging of the Physical Processes in Human Glioblastoma. Cancer Res. 74, 4622-4637 (2014).

50. Gillard, J, Waldman, A. \& Barker, P. MR spectroscopy in stroke. 168-181 (Cambridge University Press, Cambridge, UK, 2005). .

51. Ellingson, B. M. et al. Functional diffusion maps (fDMs) evaluated before and after radiochemotherapy predict progression-free and overall survival in newly diagnosed glioblastoma. Neuro. Oncol. 14, 333-343 (2012).

52. Gerstner, E., Chen, P. J., Batchelor, T. \& Sorensen, A. G. Role of diffusion MRI in the detection of infiltrative glioblastoma after treatment with Cediranib. Neuro. Oncol. 10, 894-894 (2008).

53. Wen, P. Y. et al. Report of the jumpstarting brain tumor drug development coalition and FDA clinical trials neuroimaging endpoint workshop (January 30, 2014, BethesdaMD). Neuro. Oncol. 16, vii36-vii47 (2014).

54. Best, M. G. et al. Liquid biopsies in patients with diffuse glioma. Acta Neuropathol. 129, 849-865 (2015).

55. $\mathrm{Li}, \mathrm{S}$. et al. Overexpression of isocitrate dehydrogenase mutant proteins renders glioma cells more sensitive to radiation. Neuro. Oncol. 15, 57-68 (2013).

56. Molenaar, R. J. et al. Radioprotection of IDH1-mutated cancer cells by the IDH1-mutant inhibitor AGI-5198. Cancer Res. 75, 4790-4802 (2015).

57. Romero-Garcia, S., Moreno-Altamirano, M. M., Prado-Garcia, H. \& SanchezGarcia, F. J. Lactate contribution to the tumor microenvironment: mechanisms, effects on immune cells and therapeutic relevance. Front. Immunol. 7, 52 (2016).

58. Kim, H., Thompson, R. B. \& Allen, P. S. Enhancement of spectral editing efficacy of multiple quantum filters in in vivo proton magnetic resonance spectroscopy. J. Magn. Reson. 223, 90-97 (2012).

59. Zhang, Y. \& Shen, J. Simultaneous quantification of glutamate and glutamine by J-modulated spectroscopy at 3 Tesla. Magn. Reson. Med. 76, 725-732 (2016).

60. Tkac, I. et al. In vivo $1 \mathrm{H}$ NMR spectroscopy of the human brain at 7 T. Magn. Reson. Med. 46, 451-456 (2001).

61. Dreher, W. \& Leibfritz, D. Detection of homonuclear decoupled in vivo proton NMR spectra using constant time chemical shift encoding: CT-PRESS. Magn. Reson. Imaging 17, 141-150 (1999).

62. Jafari-Khouzani, K. et al. Volumetric relationship between 2-hydroxyglutarate and FLAIR hyperintensity has potential implications for radiotherapy planning of mutant IDH glioma patients. Neuro. Oncol. 18, 1569-1578 (2016).

63. Capper, D., Zentgraf, H., Balss, J., Hartmann, C. \& von Deimling, A. Monoclonal antibody specific for IDH1 R132H mutation. Acta Neuropathol. 118, 599-601 (2009).

64. Chi, A. S. et al. Prospective, high-throughput molecular profiling of human gliomas. J. Neurooncol. 110, 89-98 (2012).

65. Dias-Santagata, D. et al. Rapid targeted mutational analysis of human tumours: a clinical platform to guide personalized cancer medicine. $E M B O$ Mol. Med. 2, 146-158 (2010).

66. Cairncross, J. G. et al. Specific genetic predictors of chemotherapeutic response and survival in patients with anaplastic oligodendrogliomas. J. Natl Cancer Inst. 90, 1473-1479 (1998).

67. Hegi, M. E. et al. MGMT gene silencing and benefit from temozolomide in glioblastoma. New Engl. J. Med. 352, 997-1003 (2005).

68. Bogner, W. et al. in Proceedings of the 23rd Annual Scientific Meeting of the ISMRM, Toronto, Canada, 30 May-5 Jun, 2015.

69. Ogg, R. J., Kingsley, P. B. \& Taylor, J. S. Wet, a T-1-Insensitive and B-1Insensitive Water-Suppression Method for in-Vivo Localized H-1-Nmr Spectroscopy. J. Magn. Reson. B. 104, 1-10 (1994).
70. van der Kouwe, A. J. W. et al. On-line automatic slice positioning for brain MR imaging. Neuroimage 27, 222-230 (2005).

71. Provencher, S. W. Estimation of metabolite concentrations from localized invivo proton NMR-spectra. Magn. Reson. Med. 30, 672-679 (1993).

\section{Acknowledgements}

We acknowledge the translational clinical oncology early drug development team at Novartis for developing the IDH305 inhibitor drug. We thank Drs. Kourosh JafariKhouzani and Morteza Esmaeili for assistance with some of the patient imaging. We thank Dr. Marjanska Malgorzata for help with the LCModel basis set for MEGA-ASER spectral editing.This work was funded by the National Cancer Institute (NCI/NIH) grants K22CA178269 and R01CA211080 (O.C.A); NIH/NCI Brain Cancer SPORE P50 CA165962 (Project 3, D.P.C.); NIH/NCI R01CA129371 and K24CA125440A (T.T.B.); NIH S10RR013026, S10RR021110, S10RR023401 (B.R.R.).

\section{Author contributions}

O.C.A., I.C.A.R., E.R.G., J.D., A.S.C., B.R.R., P.Y.W., D.P.C., and T.T.B. designed research; O.C.A., I.K.N.L., K.R., and E.M.R. performed research; W.B. contributed in the technical development of $2 \mathrm{HG}$ imaging; A.J.I. contributed molecular assays for IDH mutation; O.C.A., D.P.C., and T.T.B. analyzed data; all authors reviewed the results and wrote the paper.

\section{Additional information}

Supplementary Information accompanies this paper at https://doi.org/10.1038/s41467 018-03905-6.

Competing interests: T.T.B. received consulting honoraria from Merck \& Co., Roche, Kirin Pharmaceuticals, Spectrum, Amgen, and Novartis. The other authors declare no competing interests.

Reprints and permission information is available online at http://npg.nature.com/ reprintsandpermissions/

Publisher's note: Springer Nature remains neutral with regard to jurisdictional claims in published maps and institutional affiliations.

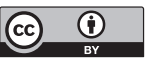

Open Access This article is licensed under a Creative Commons Attribution 4.0 International License, which permits use, sharing, adaptation, distribution and reproduction in any medium or format, as long as you give appropriate credit to the original author(s) and the source, provide a link to the Creative Commons license, and indicate if changes were made. The images or other third party material in this article are included in the article's Creative Commons license, unless indicated otherwise in a credit line to the material. If material is not included in the article's Creative Commons license and your intended use is not permitted by statutory regulation or exceeds the permitted use, you will need to obtain permission directly from the copyright holder. To view a copy of this license, visit http://creativecommons.org/ licenses/by/4.0\%

(c) The Author(s) 2018 